\title{
Protée
}

\section{De l’image cinématographique aux imaginaires télévisuels}

\section{Virginie Spies}

Volume 31, numéro 2, automne 2003

Cannes hors projections

URI : https://id.erudit.org/iderudit/008751ar

DOI : https://doi.org/10.7202/008751ar

Aller au sommaire du numéro

Éditeur(s)

Département des arts et lettres - Université du Québec à Chicoutimi

ISSN

0300-3523 (imprimé)

1708-2307 (numérique)

Découvrir la revue

Citer cet article

Spies, V. (2003). De l'image cinématographique aux imaginaires télévisuels. Protée, 31(2), 29-36. https://doi.org/10.7202/008751ar

\section{Résumé de l'article}

Comment parle-t-on du cinéma à la télévision ? Que disent les journaux télévisés du Festival de Cannes ? En cherchant à savoir comment le mode d'énonciation authentifiant de la télévision traite du Festival de Cannes, on s'aperçoit que ce sont les stars, non leurs films, qui intéressent. Depuis les actualités cinématographiques, qui insistaient sur les " vedettes » du grand écran, jusqu'à la " télé-réalité ", qui fabrique ses propres stars, il s’agit ici de mettre en évidence les spécificités discursives de la télévision en matière de cinéma. Puisant dans le réservoir à rêves cinématographique, la télévision semble imposer des règles précises, et tenter d'inscrire dans le quotidien et le réel toute la magie du $7^{\mathrm{e}}$ art. 


\section{DE L'IMAGE CIN ÉM ATO GRAPHIQ UE AU X IM AGIN AIRES TÉLÉVISU ELS}

VIRGINIE SPIES

Cannes est le lieu mystique de l'identification

de l'imaginaire et du réel. ${ }^{1}$

S'il est un lieu où l'on parle du Festival de Cannes, c'est certainement à la télévision. On en parle, on le montre, on l'expose, on le met en pâture. Ce sont des cérémonies (ouverture, fermeture), des émissions spéciales (sur les acteurs, les starlettes, les soirées), des numéros uniques, des journaux télévisés qui se penchent sur cet événement.

Il existe différentes manières de parler du cinéma à la télévision et encore plus de façons de l'étudier. Notre «ticket de cinéma» sera celui du Festival de Cannes et ses représentations, notre "part télévisuelle» sera celle du journal télévisé. Nous allons cerner ce que peut être le Festival de Cannes à travers le «mode authentifiant» à la télévision: quels aspects du cinéma sont traités? Quels thèmes sont abordés? Qui parle? Entre réalité et fiction, les questions soulevées ne permettent pas de réponses simples, mais nous promettent un parcours surprenant.

\section{LA PROMESSE DES GENRES}

Les émissions télévisuelles, indique François Jost, «sont interprétées en fonction de trois grands modes d'énonciation qui définissent à la fois l'attitude ou l'intention de celui qui adresse le document et ce que le spectateur peut en attendre ${ }^{2}$. Ces trois modes d'énonciation télévisuelle sont le mode authentifiant (regroupant les émissions tenant de vraies assertions sur le monde, c'est le cas des programmes informatifs), le mode fictif (s'appuyant sur la cohérence de l'univers créé) et le mode ludique, dans lequel se constitue un monde obéissant à ses propres codes et comprenant "aussi bien les divertissements que tous les spectacles joués" ${ }^{3}$. La reconnaissance du mode d'énonciation implique à la fois un positionnement du spectateur ainsi que des critères d'appréciation de la vérité de l'ensemble du programme. L'interprétation d'une émission dépend largement du mode d'énonciation qu'on lui attribue. On ne comprend pas de la même façon un programme selon que l'on attend de lui des informations précises ou bien que l'on 
souhaite se divertir. La question des attentes est centrale lorsqu'on se penche sur le genre.

La désignation du genre agit sur le spectateur, qui construit l'auteur. Cette construction de l'auctorialité télévisuelle par le spectateur s'accompagne d'une attente quant à son rôle ${ }^{4}$. Ainsi, les programmes s'interprètent en fonction de ce que le spectateur sait, imagine et construit de l'auteur. De plus, chaque genre porte en lui un certain type de promesse. La promesse est un acte unilatéral, qui n'oblige que le locuteur ${ }^{5}$. Les savoirs attachés aux genres sont en premier lieu fondés sur des règles constitutives. Par ailleurs,

[...] tous ces savoirs sont inégalement partagés par les téléspectateurs, et relèvent donc d'un apprentissage possible, mais ils peuvent aussi être acquis, quasi intuitivement, par une opération inférentielle. ${ }^{6}$

Ces connaissances engendrent des croyances tant sur l'émetteur que sur le monde qu'il médiatise ou construit. Ainsi, «tout genre repose sur la promesse d'une relation à un monde dont le mode ou le degré d'existence conditionne l'adhésion ou la participation du récepteur " ${ }^{7}$. Un document est sémantisé depuis son émission jusqu'à sa réception, et ne possède son statut que parce qu'il est accompagné de certaines croyances. Un document télévisuel est donc interprété à partir d'une idée que se fait le téléspectateur du type de lien qui unit ce document à la réalité. Dès lors, on peut parler de la promesse d'une relation au monde ${ }^{8}$, qui conditionne l'adhésion ou la participation du téléspectateur. Cette promesse varie selon les différents modes d'énonciation. En regardant le journal télévisé, nous sommes en droit d'attendre de vraies informations sur le monde; face à notre jeu préféré, nous pouvons espérer un certain amusement et un respect des règles de ce jeu; en assistant à notre feuilleton favori, nous sommes libres de nous évader avec le héros, d'oublier (à tout le moins) notre réalité.

Bon nombre d'émissions ne sont pas orientées vers un mode d'énonciation unique, et il est fréquent que réalité et fiction se mêlent au cours d'un même programme. De plus, le mélange de la réalité et de la fiction n'est pas un fait nouveau et, du réel au fictionnel, le discours télévisuel possède une très large palette de possibilités ${ }^{9}$. Le mélange des genres est une des particularités du discours télévisuel ${ }^{10}$.

Pour parler de cinéma, la télévision emprunte également aux différents modes d'énonciation, et c'est aussi vrai lorsqu'il est question du Festival de Cannes. Ainsi, le 1er juin 1978, un numéro de Ciné Première, intitulé "Les affaires sur les affaires», se penche, comme son titre l'indique, sur l'aspect financier du festival et des films qui y sont présentés. Sexy Cannes est une émission qui s'intéresse, le 17 mai 1990, aux starlettes et autres jolies filles qui ont arpenté les plages cannoises au cours des différents festivals. Le 11 mai 1997, une émission unique, Cannes, Les 400 coups, s'intéresse à l'histoire du festival et les événements qu'il a connus. Comme au cinéma, l'émission mensuelle consacrée au septième art, se penche, au soir du 18 mai 2000, sur l'actualité du festival, mais aussi sur les lancements des films ou encore sur les comédiens qui connaissent des difficultés. Loin d'être exhaustive, cette liste nous montre, à travers quelques exemples, autant de manières différentes de tenir un discours sur le Festival de Cannes: les questions financières, les jolies femmes, l'histoire, l'actualité, le cinéma sous son aspect factuel. Le Festival international du film aurait mille visages que la télévision peut mettre en lumière, au rythme d'émissions parfois informatives, parfois plus ludiques, souvent en mélangeant les différents modes. Enfin et surtout, chaque année, les journaux télévisés de toutes les chaînes françaises s'intéressent au Festival de Cannes.

Le journal télévisé est le genre authentifiant par excellence. Il promet de donner des informations pour améliorer notre connaissance sur le monde. Les journalistes doivent être en mesure de prouver ces informations, et l'une des manières d'attester la réalité est le direct, forme (en principe) adoptée par le journal télévisé. De plus, les individus qui s'expriment à l'intérieur du journal possèdent une légitimité (ce sont, au-delà des journalistes, des témoins, des spécialistes, ou des personnes qui concourent à 
authentifier un discours). Durant le Festival international du film, nous sommes en droit d'attendre que les journaux télévisés nous renseignent sur le festival en cours, nous informent sur ce qui s'y passe, nous éclairent sur son actualité. Un discours, fondé sur le mode authentifiant, promet de dire la ou les réalités d'un art fondé sur le fictionnel, un art qui se fête.

\section{DU RÉEL AU FICTIONNEL}

Le journal nous parle du réel. Le cinéma nous raconte des histoires inventées ${ }^{11}$. Pour que le premier parle du second, il faut un lien. On ne parle pas $d u$ cinéma. On ne s'intéresse même pas au festival, machine trop lourde pour la télévision toujours en quête de figures et d'humanité. Pour parler du Festival de Cannes, le journal télévisé a besoin d'hommes et de femmes: ce sont les stars. Du réel au fictionnel, la star est une personne «réelle» qui plonge, lorsqu'elle joue dans un film, dans le fictionnel.

Sans trahir notre corpus (essentiellement basé sur des extraits de journaux télévisés de différentes époques), un détour sous forme de retour s'impose par sa force exemplificatrice. Quittons les informations télévisées pour aller voir du côté des actualités cinématographiques. Le 31 juillet 1947, les Actualités françaises parlent d'une France d'aprèsguerre qui se reconstruit. Le dernier thème traité par ce journal est celui du Festival de Cannes et de ses préparatifs. Pour cette deuxième année du festival, on montre et on commente "l'édification du palais qui abritera la grande compétition cinématographique de l'année». Des personnalités telles que Maurice Chevalier sont sur place pour « examiner attentivement les plans». Il est déjà moins question de films que de personnes célèbres. Le 18 septembre de la même année, le journal national se penche de nouveau sur le festival. C'est la soirée d'inauguration, «de nuit, à la clarté des sunlights. C'est une soirée unique, sur la côte de l'éternel azur», indique la voix off sur des images de personnes en tenue de soirée et de stars brillant de mille feux. Cannes véhicule une image chaude et positive, c'est ce que l'on constate dans d'autres journaux de cette époque qui s'intéressent à la ville. Ajouté à cette ambiance, le festival est perçu sous un jour particulièrement positif. Après la guerre, la vie scintille de nouveau, et les figures du cinéma apparaissent comme autant d'étincelles au destin prestigieux.

Le journal national du 23 avril 1953 présente le Festival de Cannes par le biais de ses stars. On y voit une "multitude de vedettes", comme Kirk Douglas, Charles Vanel, Walt Disney. Certains font du ski nautique, d'autres se promènent en bateau ou reçoivent des prix. Si les personnes qui s'intéressaient au festival à cette époque pouvaient savoir ce qui s'y passait "réellement", il est bien plus difficile pour le chercheur, qui se pencherait uniquement sur ces sources cinquante ans plus tard, de savoir quels films concouraient, quels étaient les débats ou les thèmes que le cinéma abordait alors. Les journaux parlaient des vedettes, en aucun cas des films. Cette constatation se confirme l'année suivante, avec des images et des textes entièrement consacrés à Robert Mitchum, Michèle Morgan et d'autres «vedettes». Le commentaire distingue d'ailleurs deux types de vedettes: les "vedettes officielles", que sont le ministre d'État, le directeur du centre du cinéma, ou encore le président du festival, Jean Cocteau, et les «vedettes de l'écran", que sont les acteurs. Lors de la clôture de ce festival, il s'agit même de montrer et de commenter le repas que «les vedettes» ont dégusté pour «reprendre des forces». Ce sont encore les stars dont il est question dans le journal du 21 mai 1958, qui met en avant «les invités de la dernière heure». Au-delà des stars, on évoque les rumeurs qui les concernent. À la fin des festivités, quelques phrases résument, en conclusion de reportage, le palmarès du festival.

Les actualités cinématographiques parlent du Festival international du film à travers les vedettes (c'est d'ailleurs le terme le plus souvent employé). Ces observations rejoignent celles d'Edgar Morin: «À Cannes, ce ne sont pas tant les films que les stars qui s'exhibent en spectacle" ${ }^{12}$. Ainsi, ajoute-t-il, le festival est avant tout (en tout cas pour la presse) le rendezvous des stars. Les actualités en sont effectivement le 
reflet. Les stars posent «pour l'univers entier par le truchement de la photographie, de la télévision et des actualités» ${ }^{13}$. On parle de ces stars, mais on ne leur donne pas encore la parole. Tout d'abord parce que la forme des actualités cinématographiques ne s'y prête pas, ensuite parce que les stars ne possèdent pas encore le statut qu'elles vont acquérir par la suite, et qui fera qu'elles auront la parole lors de chaque reportage qui sera consacré à leur film. Pour l'heure, la star prend la pose, l'air penseur, cheveux au vent.

\section{LA VOIX DES STARS}

Avec la télévision, les stars continuent de poser sur la plage, mais elles vont aussi s'exprimer. Partout, sur tout, à tout moment. À une exception près, lorsque les membres du jury viennent de rendre leur verdict, en ce 25 mai 1977, et que Léon Zitrone tente de les interpeller pour leur demander des informations sur leurs choix. Ce jury, bien entendu composé de stars (avec Rossellini comme président), va rester muet jusqu'à ce que le verdict soit rendu.

À Cannes, ce sont les noms qui comptent, bien plus que les films. Ainsi, lorsque Bernard Rapp, alors présentateur du journal télévisé sur Antenne 2 en ce mois d'avril 1985, donne la sélection française pour le festival, il n'indique aucun titre, aucun genre, mais bien des noms d'auteurs. Godard, Chabrol et Téchiné suffisent à donner le ton de la sélection.

Le 15 mai 1990, Patrick Poivre D'Arvor (autre vedette, mais du journal de 20 heures sur TF1) «lance» un reportage sur l'actrice Sandrine Bonnaire: tout à son rôle de star, elle signe des autographes, "court de rendez-vous en rendez-vous", parle d'elle et de ses goûts. Du point de vue filmique, le reportage télévisé plonge le téléspectateur dans un univers de cinéma entièrement consacré aux stars: ralentis et musique sont autant de signes qui se reportent au septième art et qui sont assez peu usités dans le discours télévisuel. Le sujet et son traitement permettent même au présentateur d'assurer une transition pour le reste de la soirée sur la chaîne TF1, puisqu'il va y avoir un film, avec «d'autres vedettes». Désormais, les stars «appartiennent» autant au cinéma qu'à la télévision. Au-delà de la promotion, la télévision a d'autres rôles: elle finance le cinéma, elle fait exister les stars (comment peut-on «être connu» si le petit écran ne s'intéresse pas à nous?), elle les fait travailler. En effet, nombre de stars françaises ont joué dans des fictions télévisuelles: Gérard Depardieu, Jean-Paul Belmondo, Carole Bouquet en sont des exemples. Le même jour, sur la chaîne concurrente, Antenne 2, Philippe Lefait parle également de Sandrine Bonnaire en termes élogieux. Le film dans lequel on peut la voir est réalisé par Raymond Depardon, dont on va faire le portrait. On insiste sur la personnalité et la vie du photographe et réalisateur. Il est à peine question du film qu'il présente. La télévision semble incapable de parler de produits culturels; elle se penche sur les acteurs de ces produits culturels. Le lendemain, le journal de la même chaîne propose plusieurs sujets et interviews, entièrement tournés vers les stars du festival. Un reportage est consacré à un enfant qui est un chasseur d'autographes, c'est «une autre façon d'approcher les vedettes». Le Festival international du film est assez mal nommé. Il s'agit plutôt du festival des stars. Le 21 mai de la même année, on remet la Palme d'or. À écouter Hervé Claude, alors présentateur du journal de 20 heures sur Antenne 2, ce n'est pas le film Sailor et Lula qui est couronné, mais plutôt David Lynch. Le discours télévisuel ne donne aux films aucune existence ni droit de citation, s'ils ne sont pas rattachés à leurs différents auteurs. Le même jour, sur TF1, le discours est le même. Patrick Poivre D'Arvor explique que la Palme d'or est «décernée par le jury à l'Américain David Lynch, l'auteur d'Elephant man et de Blue Velvet». La Palme d'or n'est donc pas décernée à un film, mais à un homme. La même année, le prix d'interprétation est remis à Gérard Depardieu pour son rôle dans Cyrano de Bergerac. L'acteur est félicité et fêté par les deux chaines de télévision. On rend hommage aux Hommes qui ont fait un film. Ce soirlà, on atteint le paroxysme avec cette phrase du présentateur de TF1, qui dit en fin de journal: «Voilà donc pour ce très beau Cyrano de Rappeneau, de Depardieu, et bien sûr, de Rostand». 
De la même manière, est présenté, lors du festival de l'année 2000, le film Saint Cyr qui met en scène Isabelle Huppert dans le rôle de Madame de Maintenon. Sur France 2, le 16 mai, un reportage est entièrement consacré à deux jeunes filles qui ont tenu des rôles importants dans le film. Le commentaire explique que ce sont "deux inconnues confrontées à une star (Isabelle Huppert) du jour au lendemain». La star est en duplex, à Cannes, et elle est interviewée par le présentateur du journal qui ne va pas lui parler de son travail d'actrice ou des conditions de tournage, mais plutôt de la manière dont elle perçoit la dernière épouse de Louis XIV. Il s'agit de savoir «ce qui l'a le plus intéressé chez Madame de Maintenon ». On lui demande aussi: «qu'est-ce qui vous a semblé le plus proche de vous chez ce personnage?». Il n'est pas question de la réalisation du film, il s'agit d'une star dont tous les propos peuvent être dignes d'intérêt. Dans la même semaine et sur la même chaîne, Béatrice Schönberg, qui présente le journal le weekend, parle de Cannes comme d'«une immense machine à rêves, avec ses stars et ses palaces". La même semaine encore, sur TF1, il est question de «brochette d'acteurs prestigieux et internationaux».

Des territoires empruntés ou créés par le journal télévisé pour parler du Festival de Cannes, celui des stars et de leur vie publique ou privée est le plus prisé. Le discours télévisuel personnifie. Ce qui compte, ce sont les Hommes: auteurs ou acteurs. Cannes est représenté comme un lieu où les stars se retrouvent, et où les films n'ont pas d'existence propre. Le journal télévisé informe très peu, il se penche, ou penche vers d'autres lieux, territoires étoilés. Ce phénomène nous montre l'omniprésence de la télévision dans le monde du cinéma. Partout, à chaque moment, les stars sont là pour parler d'elles, de leur vie, de leurs récompenses. Leur opinion a de la valeur, que ce soit à propos d'un personnage historique ou de leur divorce à venir (la vie privée d'une star se doit d'être publique, indique Edgar Morin). Il s'agit d'un être là, qui accentue la valeur d'une parole qui n'a d'intérêt que parce qu'elle est prononcée par quelqu'un de "connu». La visibilité confère la légitimité.
«Les stars mènent une vie de festival: le festival mène une vie de stars - une vie de cinéma" ${ }^{14}$. Du point de vue des images, on privilégie les gros plans, les ralentis et même la musique. Discours télévisuel au service de la star: divine et mythique, elle appelle à une esthétique spécifique. Lorsque les reportages ne se penchent pas sur des stars, il en est tout de même question (c'est une star en devenir, c'est quelqu'un qui connait des stars, ou qui aimerait beaucoup en connaître, un reportage sur les femmes de chambre du Carlton, un chasseur d'autographes, des spectateurs lambda qui attendent, des heures durant, aux marches du palais).

Le cinéma raconte des histoires. La télévision qui parle de cinéma raconte les histoires des gens de cinéma. En accédant au journal télévisé, les stars entrent dans le quotidien du téléspectateur, à la frontière de l'accessible et de l'inaccessible. Au-delà de leur mythe, les stars se veulent plus proches du public, et la télévision est un moyen d'y accéder. Point de contact entre la star et son public, le petit écran permet à la star d'entrer dans son salon. Ce qui compte est moins ce qui est dit que la personne qui le dit. Le sujet de l'énonciation est bien plus important que l'énoncé.

Et s'il était impossible, pour la télévision, de parler de cinéma autrement? La mythologie des stars, indique Edgar Morin, «se situe dans une zone mixte et confuse, entre croyance et divertissement " ${ }^{15}$. Il s'agit bien du lien auquel nous faisions référence précédemment. La star est ici le ciment du réel et du fictionnel.

\section{LE MODE AUTHENTIFIANT AU SERVICE DU RÊVE}

Mais justement, qu'en est-il du réel? Le journal télévisé, nous l'avons vu, est censé restituer le réel, dire le monde au téléspectateur.

Le Festival de Cannes débute avec l'après-guerre, l'heure est à la fête et à la distraction. Le cinéma pour oublier le réel. Ce que représente le cinéma, ce n'est pas ce qu'Est le cinéma. Les informations télévisées restituent une part du réel. C'est ce que le téléspectateur est en droit d'attendre. Il s'agit ensuite 
d'accentuer le réel, ce qu'on va faire en mettant en lumière la star.

Très importante également est la valeur marchande des films et des stars. Ces dernières sont des marchandises qui s'exposent sur le grand marché du visuel. Elles font la promotion de leur film. Mais y aurait-il de meilleurs vendeurs? Qu'on s'identifie à l'acteur ou que, plus simplement, on l'apprécie, il est un bon «prescripteur». La star est un objet de consommation, qui, au-delà de la "vente» de son film, doit exister sur le petit écran pour exister aux yeux du public. Avec la rapidité de circulation de l'information et des images, "la dimension mythique des autres s'efface " ${ }^{16}$. Un rapport de proximité s'installe et nous avons vu que, pour être star, le mythe a désormais besoin d'être ravivé souvent. La télévision en est un moyen. "Les vedettes ne tiennent plus longtemps l'affiche, les nouvelles "révélations" éclipsent celles d'hier selon la logique de la personnalisation " ${ }^{17}$. Il y a, pour Gilles Lipovetski, de plus en plus de «vedettes", mais elles suscitent de moins en moins

d'investissement émotionnel. Cette logique de la personnalisation permet plus de s'accomplir soi-même que de s'intéresser aux autres. Cet intérêt pour ces "autres», les stars de cinéma, permet de tenir un discours minimal mais nécessaire sur le cinéma.

De plus, les interventions des acteurs sont autant de consignes de lecture qui sont susceptibles de jouer sur la réception du film. Les propos des stars agissent sur le genre, et l'étiquette générique apposée influence la réception. Les stars qui commentent leur film sont inscrites dans un contexte, qui lui aussi donne une identité au produit qu'est le film. En elles-mêmes, certaines stars déterminent le genre de film que le spectateur s'attend à voir, elles confèrent une identité première, une promesse. On s'attend plutôt à une comédie romantique avec Meg Ryan, à un film d'aventure avec Bruce Willis, etc.

«L'auteur est ce qui donne à l'inquiétant langage de la fiction, ses unités, ses nœuds de cohérence, son insertion dans le réel", écrit Foucault ${ }^{18}$. Par la force de ce qu'une star peut imprimer à un film, elle est à considérer comme une forme d'auteur. À la fois en dehors du film et en dedans, elle assigne à son personnage et à son film un caractère qui ressortit au réel. On comprend mieux alors pourquoi les journalistes interrogent plus les acteurs sur leur rapport au réel (que pense Isabelle Huppert de Madame de Maintenon?), que sur leur rapport à la fiction.

\section{UNE IMPOSSIBLE CRITIQUE}

$\mathrm{Si}$, à propos du Festival de Cannes et des films en compétition, les journaux télévisés informent peu, ils ont encore moins la capacité d'émettre une quelconque critique.

Les films sont souvent "d'une force incroyable». À propos de Dancer in the dark lors du festival de l'année 2000, il est question sur France 2 d'un film «original et novateur", c'est "un très, très beau triomphe, bien mérité». TF1 parlera, à propos de la même œuvre, d'un «film très fort, très émouvant». Sur France 2, on trouve «Isabelle Huppert bouleversante» dans Saint Cyr. Sur TF1, le film de Raymond Depardon «force le respect». Sur France 2 encore, Sandrine Bonnaire est remarquable. Le plus souvent, les stars présentes «en plateau " sont au service de la notoriété de la chaine ( on remercie Catherine Deneuve de nous faire l'amitié de sa présence» dit Claire Chazal le 21 mai 2000). De plus, il s'agit, dès que c'est possible, de saluer le cinéma français.

Les présentateurs félicitent les stars qui se congratulent entre elles. En demandant leur avis à celles qui ont tourné dans le film, on ne peut espérer obtenir un regard critique sur l'œuvre. Plus légitime serait la parole du scénariste, du réalisateur. Plus critique serait la parole du journaliste de cinéma. D'une parole forte, le critique se positionne comme «un messager de l'opinion, comme le représentant du pouvoir du public» ${ }^{19}$. Mais le journal télévisé ne véhicule pas ce type de discours. La critique est utopique puisqu'elle suppose un ailleurs, «un monde fictionnel meilleur». Le journal télévisé glorifie et encense, il ne laisse pas de place pour la critique des films, ou la critique du choix des récompenses. Le journal télévisé n'en est pas le lieu. De plus, c'est à la 
fin du journal qu'il est question de cinéma. Avant la publicité, le ton se veut plutôt léger et agréable. En effet, il ne faut pas omettre les spécificités du médium...

\section{UNE MACHINE À FABRIQUER DES «STARS»?}

Les mises en scène du quotidien des Français sont fréquentes à la télévision et trouvent leurs racines dans des programmes produits dès les années 195020 . L'importance du quotidien est vraisemblablement inhérente au médium lui-même (par la place qu'il occupe dans le quotidien des téléspectateurs) et aux formes de discours qu'il met en œuvre (il s'agit ici de l'intérêt porté par le médium aux téléspectateurs euxmêmes).

Si la télévision des années 1960 «se servait» des stars du cinéma, si elle le fait encore aujourd'hui pour alimenter son discours sur le cinéma, on remarque que, désormais, elle fabrique des stars en dévoilant plus ou moins le processus de fabrication. Depuis 2001, des chaînes privées du réseau herztien français diffusent des programmes de ce type. Il s'agit de Star Academy sur TF1 et de Pop Stars sur M6. Différents dans leurs principes mais semblables dans leurs finalités, ces programmes, souvent qualifiés de "téléréalité» ${ }^{21}$, partent de personnes inconnues, les rendent célèbres (grâce à leur présence quotidienne ou hebdomadaire sur la chaîne) et vont ensuite, tant que leur succès durera, suivre ces personnes, nouvelles stars, dont bien souvent le mérite principal est d'avoir été «vues à la télé». Tirée de son quotidien, la nouvelle star alimentera les discours médiatiques autour de sa nouvelle vie et de son nouveau quotidien.

La première émission française de ce type, Loft Story, est apparue au printemps 2001 sur M6. Devenus stars de l'instantané, les premiers «lofteurs» à sortir de leur prison $d u$ visuel se rendirent au Festival de Cannes... Des stars de cinéma à la télévision, nous sommes passés aux stars de la télé dans des lieux de cinéma.

Vedettes fabriquées par le petit écran ou acteurs de cinéma, les stars viennent à Cannes pour se donner en spectacle, c'est un fait que Jean-Luc Godard, par exemple, a bien compris ${ }^{22}$. Le 10 mai 1985, Daniel Bilalian parle du cinéaste dans son journal, car il a reçu une tarte à la crème en pleine figure. "À Cannes, au festival, la réalité, c'est du cinéma», dit-il. Un peu plus tard, Jean-Luc Godard dira que ce sont la presse et les médias qui font l'événement à Cannes. Qui pourra le contredire?

En essayant de comprendre une partie de ce qu'est le festival, par l'une de ses représentations, celle qui est donnée par le mode authentifiant à la télévision, nous avons observé que le cinéma est toujours rattaché à des Hommes. Le cinéma est la vie et la télévision, avec ses spécificités, parle peu du réel mais choisit certaines formes de réalités: celles qui sont attachées aux personnes qui font du cinéma. Le mode authentifiant est un cadre à l'intérieur duquel il est possible de composer plusieurs types de discours. Jean-Marie Schaeffer ${ }^{23}$ a montré l'importance de la fonction de divertissement dans la fiction. Les stars sont là pour divertir le public. Dès lors, dans le journal télévisé, elles sont à la frontière des trois modes d'énonciation principaux à la télévision que sont le mode fictif, le mode authentifiant et le mode ludique. En effet, la star est en premier lieu sortie de la fiction pour être une personne à laquelle on va demander son avis, ses sentiments, etc. La perception de la star est pourtant, nous l'avons vu, dépendante de ses films, et elle poursuit une représentation qui a pris forme dans le monde fictionnel. Elle pénètre donc dans l'univers du réel, c'est le mode authentifiant, attesté par la forme du journal télévisé. Dans ce mode se crée un monde dans lequel sont tenues de vraies assertions qui permettent d'améliorer nos connaissances de ce monde. Le journal télévisé et le direct concourent à la construction de ce mode. Enfin, on aborde le sujet du cinéma et celui de la star au terme des journaux télévisés, lorsqu'il est temps de quitter les informations internationales, politiques ou sociales, pour entrer dans un univers plus ludique (ce qui permet d'assurer une transition avec la publicité à 
venir). C'est vers ce mode ludique que penche en dernier lieu le discours autour de la star. Il est effectivement question d'un discours de vérité, avec un système de règles particulières, des glissements possibles et même souhaitables et un ton caractéristique bien moins sérieux que lorsqu'il est question d'informations plus graves. La vérité du discours télévisuel se juge en fonction des genres qui «se définissent comme la promesse d'une relation à un monde "24. Les programmes analysés ici se saisissent à partir du mode authentifiant, qui est sensible aux autres modes d'énonciation - apportés par le sujet dont il est question et la manière dont il est traité.

Entre réel et fictionnel, Edgar Morin se penche sur l'image: «L'écran paraissait devoir présenter un miroir à l'être humain: il offrit au XXe siècle ses demi-dieux, les stars " 25 . Du grand au petit écran, la frontière est de plus en plus floue et le regard, susceptible de se brouiller lorsqu'il tente de contempler le miroir.

\section{NOTES}

1. E. Morin, Les Stars, Paris, Éd. du Seuil, coll. «Points», 1972, p. 57.

2. F. Jost, La Télévision du quotidien: entre réalité et fiction, Bruxelles, De Boeck Université, Paris, INA, coll. «Médias-recherches », 2001, p. 17.

3. F. Jost, "Quand y a-t-il énonciation télévisuelle?", dans J. Bourdon et $\mathrm{F}$. Jost (sous la dir. de), Penser la télévision, Actes du colloque de Cerisy, Paris, Nathan et INA, coll. "Médias-recherches", 1998, p. 35. 4. Ibid., p. 42.

5. F. Jost, «La promesse des genres», Réseau, nº 81 (Le genre télévisuel), 1997.

6. Ibid., p. 19.

7. F. Jost, La Télévision du quotidien, p. 17.

8. Ibid.

9. Ibid.

10. Voir sur cette question V. Spies, «Un drôle de regard réflexif: quand les Guignols de l'info parlent du PSG", dans P. Gabaston et B. Leconte, Sports et Télévision, Paris, L'Harmattan, coll. «Communication et Civilisation", 2000, ainsi que "Les Guignols de l'info - Le mélange de genres à son paroxysme", dans Actes du Colloque d'Aix-enProvence, mai 2000, à paraître.

11. Il faut reconnaître qu'il arrive que le journal télévisé raconte des histoires et que le cinéma nous parle du réel, mais ce ne sont pas les règles habituelles de leur discours.

12. E. Morin, Les Stars, p. 56.

13. Ibid., p. 58.

14. Ibid., p. 57-58.

15. Ibid., p. 8 .

16. M. Augé, Pour une anthropologie des mondes contemporains, Paris, Flammarion, coll. «Champs", 1997, p. 26.

17. G. Lipovetski, L'Ėre du vide. Essais sur l'individualisme contemporain, Paris, Gallimard, coll. «Folio », 1989, p. 105-106.

18. M. Foucault, L'Ordre du discours, Paris, Gallimard, 1971, p. 30.

19. P. Schaeffer, Critères et fonctions de la critique de la télévision, Actes du congrès de Turin, 1972, p. 88.

20. F. Jost, La Télévision du quotidien, p. 109-200.

21. Voir à ce sujet F. Jost, L'Empire du loft, Paris, La Dispute, coll. «Des mots sur les images", 2002.

22. Voir à ce sujet F. Jost, "Au nom des pairs: Godard à Cannes", dans E. Ethis (sous la dir. de), Aux marches du palais. Le Festival de Cannes sous le regard des sciences sociales, Paris, La Documentation française, 2001.

23. J.-M. Schaeffer, Pourquoi la fiction?, Paris, Éd. du Seuil, coll.

"Poétique», 1999.

24. F. Jost, La Télévision du quotidien, p. 36.

25. E. Morin, Les Stars, p. 7. 